\title{
Estradiol Inhibition of Catecholamine Elicited Eating in the Female Rat
}

\author{
C. WAYNE SIMPSON AND LEO V. DICARA \\ Mental Health Research Institute, Department of Psychiatry \\ The University of Michigan, Ann Arbor, Michigan 48104
}

(Received 26 April 1973)

\begin{abstract}
SIMPSON, C. W. AND L. V. DICARA. Estradiol inhibition of catecholamine elicited eating in the female rat. PHARMAC. BIOCHEM. BEHAV. 1(4) 413-419, 1973.-Two experiments test the hypothesis that estradiol suppresses food intake by inhibiting $\beta$ dopamine hydroxylase in the anterior diencephalon where norepinephrine sensitive neurons activate feeding behavior. In the first experiment, dopamine or norepinephrine was injected intracerebrally in female rats during estrous and diestrous phases of the estrous cy cle. Dopamine injections in the anterior diencephalon of rats elicited eating $(p<0.05)$ only during diestrus when circulating estradiol is low; norepinephrine elicited eating during both diestrous $(p<0.02)$ and estrous $(p<0.001)$ phases of the cycle. In the second experiment the catecholamines were administered to ovariectomized rats with and without estradiol replacement. Again dopamine was effective in eliciting food intake $(p<0.05)$ only in the absence of circulating estradiol while the increases following norepinephrine were independent of estradiol treatment.
\end{abstract}

Estradiol inhibition Catecholamine eating Anterior diencephalon

Intracerebral injections $\beta$ dopamine hydroxlase

SEVERAL investigators have recently demonstrated the inhibitory influence of the steriod sex hormone $17 \beta$ estradiol on food intake and body weight regulation $[8,9,14$, $16,17,19,20]$. Possible mechanisms of action proposed to account for the estradiol inhibition have included a lowering of the body weight set point mediated at an unspecified central locus $[8,19]$, or increased $\mathrm{VMH}$ activity which inhibits LH feeding responses, and/or the induction of finicky eating behavior due to the action of circulating estradiol on the ventromedial nucleus of the hypothalamus [17].

As a result of neuroendocrine studies demonstrating changes in regional brain catecholamine levels following manipulation of estradiol titers and Booth's [1] report of the anatomical distribution of catecholamine elicited feeding sites, an alternative hypothesis concerning the mechanism of estradiol inhibition, was investigated in the senior author's dissertation research [10]. A brief review of the neuroendocrine results is necessary before the hypothesis is described.

Donoso and Stefano [2], observed significant norepinephrine (l-NE) increases and reciprocal dopamine (DA) decreases in the anterior hypothalamus of female rats following bilateral ovariectomy. This effect was not observed in posterior hypothalamus or cortex. High daily doses of exogenous estradiol benzoate and progesterone reversed the l-NE and DA relationship in the anterior hypothalamus to that observed in intact rats. Donoso and Cukier [3], used groups of animals under different endocrine conditions of estradiol influence which were followed by ovariectomy. In all groups high levels of estradiol were associated with very low levels of $1-\mathrm{NE}$ in the anterior hypothalamus. However, following ovariectomy, 1-NE levels increased significantly in anterior hypothalamus. In the normally cylcing female rat $1-\mathrm{NE}$ levels in anterior hypothalamus are very low at estrus and increase during diestrus [12]. Increased turnover rates for $1-N E$ in rat anterior hypothalamus following ovariectomy have also been reported $[4,15]$.

In his investigation of the distribution of catecholamine sites in rat brain, Booth [1] noted that the most intense eating following a $1 \mu \mathrm{l}$ injection of $1-\mathrm{NE}$ occurred in and around the interstitial nucleus (INST) of the stria terminals dorsal to the anterior hypothalamic area in the rostral portion of the rat brain. Stumpf [13] in an elegant paper tracing the distribution of estrogen concentrating neurons in the female rat brain identifies the interstitial nucleus of the stria terminals as an anatomical locus with a very high density of estrogen sensitive neurons. The overlapping distributions of catecholamine sensitive and estrogen sensitive neurons from the studies of Booth [1] and Stumpf [13] suggested the hypothesis that the mechanism of the inhibitory action of estradiol on food intake and body weight was a biochemical interaction at specific anterior hypothalamic loci. Specifically, it was hypothesized that

\footnotetext{
'Experiment 1 of this article is based on a dissertation submitted to the Graduate School of the University of California Santa Barbara in partial fulfillment of the requirements for the degree, Doctor of Philosophy. The senior author is very grateful to Professors Robert W. Reynolds, H. J. Carlisle, and Russel A. Lockhart for their advice and encouragement. The research was supported by USPHS Predoctoral Fellowship 4Fol GM 37720-03 to C. Wayne Simpson and Grant No. 21403 to Leo V. DiCara.
} 
estradiol inhibits $\beta$ dopamine hydroxylase, thereby disrupting the conversion of dopamine to norepinephrine at specific anterior hypothalamic loci. This proposed mechanism predicts that DA should elicit significantly increased eating whenever estrogen levels are sufficiently low to permit the conversion of DA to 1-NE. On the other hand, l-NE should produce significant increases in food intake regardless of estrogen levels since 1-NE is beyond enzymatic inhibition. This hypothesis was tested in the two experiments reported herein by (1) injecting l-NE and dopamine into anterior hypothalamus and other brain locations in normally cycling female rats and (2) by hypothalamic injections of l-NE and dopamine into the INST area of female rats following ovariectomy and exogenous estrogen treatment.

\section{EXPERIMENT 1}

The general design of Experiment 1 was the injection of l-NE and DA $\mathrm{HCl}$ into specific brain sites during estrous and diestrous phases of the natural estrous cycle in rats and the consequent effects on food intakes.

\section{Method}

Animals. Sixty-six Sprague-Dawley female rats (BerkleyPacific) served as subjects. Rats were 90 days old at the time of surgery. Only females displaying 4 or 5 day estrous cycles were used for the experiment.

Surgery. Bilateral cannulas were prepared as described in detail elsewhere [10] and implanted in all 66 female rats. Individual guideshafts were stereotoxically placed $1 \mathrm{~mm}$ above each of the following brain loci: Interstitial nucleus of the stria terminalis, and anterior hypothalamus, forming an anterior diencephalic group (AD); in midthalamus to sample sites in middiencephalon (MD); and in the prerubal Fields of Forel for posterior diencephalic sites (PD). MD and PD sites were selected because they do not demonstrate catecholamine elicited eating, nor take up circulating estradiol. Rats recovered on ad lib food and water for one week following surgery.

\section{Feeding Schedule}

Following recovery from surgery all rats were maintained on a daily satiation feeding schedule described by Booth [1]. This schedule consisting of 10 min access to wet mash, 30 min access to lab chow pellets (Purina), followed by an additional $60 \mathrm{~min}$ of lab chow pellets. Injections were delivered at the end of the 30 , but before the $60 \mathrm{~min}$ component. At the end of the $60 \mathrm{~min}$ component a preweighted number of pellets were left in the home cage overnight, and the remainder weighed the following morning to determine daily food intakes. Booth [1] has described this feeding schedule as particularly sensitive to l-NE elicited changes in food intake.

Water was weighed immediately before and after the feeding series and also on the following morning to determine $24 \mathrm{hr}$ intakes. Following the morning weighing of food rats were deprived of all food for approximately $5 \mathrm{hr}$ between the morning weighing and the afternoon injection. After 3 weeks training and 5 sham (i.e., cannula inserts connected but nothing injected) injections using the satiation procedure, catecholamine injections were begun. The feeding interval following DA injections was extended to 120 min since Slangen and Miller [11] reported that DA injections reliably elicited eating during the second but not the first hour after injection.

\section{Injection Procedures}

Injection procedures were the same for all animals: 2 $10 \mu \mathrm{l}$ Hamilton syringes filled with sterile $0.9 \%$ saline dopamine hydrochloride, or l-norepinephrine bitartiate were connected with polyethelene tubing (PE20) to individual cannula inserts, which extended $1 \mathrm{~mm}$ below the lumen of the guideshafts into the target tissue, and $1 \mu 1$ was injected bilaterally. l-NE bitartiate $(20 \mu \mathrm{g} / \mu \mathrm{l})$ and dopamine $\mathrm{HCl}(100 \mu \mathrm{g} / \mu \mathrm{l})$ solutions were made up daily in sterile saline $0.9 \%$.

Both catecholamines and sterile saline were injected in a counterbalanced order, during both estrous and diestrous phases of the cycle for a total of 16 injections. The 16 injections consisted of $4 \mathrm{NE}, 4$ dopamine and 8 interspersed saline injections.

\section{Determination of Estrous State}

Estrous and diestrous conditions were determined from daily vaginal smears examined microscopically. The classic description [18] of the estrous smear featuring a large number of cornified epithelial cells and the absence of leucocytes was taken as the criterion for estrous. Diestrous was classified by the absence of cornified epithelial cells with a large number of leucocytes. All slides which did not fulfill the criterion of an estrous smear were considered to be diestrous.

Since an estrous day occurred every 4 or 5 days the catecholamine injections were necessarily discontinuous. Diestrous injections were given daily in counterbalanced order until an estrous day intervened. When a rat completed the 8 injections in the diestrous phase it was then injected only on estrous days, usually once every 4-5 days, following the counterbalanced order of saline and catecholamine injections until all injections in the estrous phase of the cycle were completed.

\section{Histology}

Following the final injection rats were overdosed with numbutal and perfused. Brains were cut at $20 \mu$ and stained with cresyl violet for microscopic localization of cannula.

\section{Results}

Several animals died as a result of intracranial hemorrage during the experiment. The $\mathrm{N}$ column in Table 1 represents only rats which completed a complete injection sequence, i.e., of 1-NE or DA-HCl for the particular endocrine condition noted for that group. All data were analyzed by the $t$-test for correlated observations. Table 1 shows the mean differences in food intakes following l-NE and dopamine $\mathrm{HCl}$ injections compared to saline injections in the same rats during both estrous and diestrous phases of the normal cycle for all of the anatomical groups used in this experiment. As predicted, l-NE injections during either estrous or diestrous elicit significant increases in food intake; estrous $(p<0.001)$ and diestrous $(p<0.02)$ in the anterior diencephalic groups. Also, as predicted, DA $\mathrm{HCl}$ injections elicited significant increases in food intake only during the diestrous phase $(p<0.05)$ of the estrous cycle. Food intake following dopamine injections during estrous did not differ 
TABLE 1

MEAN DIFFERENCES IN FOOD INTAKE FOLLOWING 1-NOREPINEPHRINE AND DOPAMINE HCI INJECTIONS FOR ALL ANATOMICAL GROUPS DURING ESTROUS AND DIESTROUS

\begin{tabular}{|c|c|c|c|c|c|c|c|c|}
\hline & \multicolumn{4}{|c|}{ Estrous } & \multicolumn{4}{|c|}{ Diestrous } \\
\hline & $\begin{array}{c}\text { Mean } \\
\text { Difference }\end{array}$ & $\mathbf{N}$ & S. D. & $p$ & $\begin{array}{c}\text { Mean } \\
\text { Difference }\end{array}$ & $\mathbf{N}$ & S. D. & $p$ \\
\hline \multicolumn{9}{|c|}{ Norepinephrine } \\
\hline Ant. Dien. & +3.38 & 26 & 0.78 & $<0.001$ & +1.45 & 21 & 0.67 & $<0.02$ \\
\hline Mid. Dien. & +0.58 & 12 & 0.50 & $>0.05$ & +0.09 & 12 & 0.20 & $>0.05$ \\
\hline Post. Dien. & +0.78 & 9 & 0.54 & $>0.05$ & -0.17 & 9 & 0.56 & $>0.05$ \\
\hline \multicolumn{9}{|l|}{ Dopamine } \\
\hline Ant. Dien. & -0.07 & 19 & 0.56 & $>0.05$ & +0.97 & 15 & 0.50 & $<0.05$ \\
\hline Mid. Dien. & +0.09 & 12 & 0.02 & $>0.05$ & +0.79 & 12 & 0.41 & $>0.05$ \\
\hline Post. Dien. & +0.44 & 8 & 0.57 & $>0.05$ & +0.80 & 5 & 0.20 & $<0.01$ \\
\hline
\end{tabular}

significantly $(p>0.05)$ from food intakes following saline injections.

I-NE injections during either estrous or diestrous did not affect the food intakes of rats implanted in the mid or posterior diencephalon. Dopamine $\mathrm{HCl}$ injections, on the other hand, did elicit a significant increase in food intake $(p<0.01)$ in the posterior diencephalic group during diestrus. The mid diencephalic animals were unresponsive to DA $\mathrm{HCl}$ injections during either phase of the estrous cycle.

\section{Histology}

Figure 1 is a photograph of a stained brain section showing an example of cannula tips located in the (INST) for one rat from this experimental group. All rats showed very similar patterns of cannula localization and tissue damage. Both poles are located in the confluence of the (INST) approximately parallel to the top of third ventricle about $1.5 \mathrm{~mm}$ lateral and between $2.0-3.0 \mathrm{~mm}$ dorsal to the ventral surface of the brain.

Figure 2 shows a schematic representation using figures from the König and Klippel [6] atlas of the rat brain to identify the other brain regions sampled in this experiment. The plates indicate a sample of cannula placements in the anterior hypothalamus, midthalamic area, and perubral area. All injection sites were localized anatomically by reference to the atlas of the rat diencephalon.

\section{EXPERIMENT 2}

Due to the remarkable agreement between the obtained feeding results and the experimental predictions regarding the direction of feeding effects following catecholamine injections during the natural estrous cycle it was deemed necessary to conduct a critical experiment which would allow independent manipulation of both catecholamine injections and the hormone level. Since only the rats with anterior diencephalic cannulas showed systematic changes in feeding behavior during the natural estrous cycle following 1-NE or DA $\mathrm{HCl}$ injections, all rats in Experiment 2 had cannulas aimed for this area.

\section{Method}

Animals. Eighteen female rats of the Sprague-Dawley strain (Spartan) 90 days old at the time of surgery were used in Experiment 2.

Surgery. All animals were initially implanted stereotaxically with bilateral cannulas in the intestitital nucleas of the stria terminalis described in Experiment 1. Rats were allowed a post operative period of two weeks on ad lib food and water. Rats were then anesthetized again with Equithe$\sin (0.33 \mathrm{cc} / 100 \mathrm{~g}$ body weight) and bilaterally ovariectomized. Ovariectomy consisted of complete removal of the ovary and oviducts and approximately $1 / 3$ of the uterine horn bilaterally. Animals recovered for one week on ad lib food and water following ovariectomy.

\section{Feeding Schedule}

For the next two weeks all animals were weighed daily and maintained on the feeding schedule described in Experiment 1 . Sham injections occurred the last four days of the third week following ovariectomy. A period of three weeks between ovariectomy and catecholamine injections was instituted to allow for the complete disappearance of estradiol.

\section{Injection procedures}

Systemic. Exactly 21 days following ovariectomy the 18 rats were divided into 3 groups of 6 animals each. Rats in Group 1 received catecholamines but no systemic injections, Group 2 rats were injected with catecholamines and systemically with sesame oil $(0.2 \mathrm{ml})$, Group 3 rats received 1-NE and DA injections centrally and systemic injections of sesame oil mixed with $17 \beta$ estradiol $(50 \mu \mathrm{g})$, also $0.2 \mathrm{ml}$. All systemic injections were given daily subcutaneously immediately after the morning weighing for 8 consecutive days.

Central. 1-NE, dopamine $\mathrm{HCl}$, and sterile saline $0.9 \%$ were injected intracerebrally in all groups daily in the counterbalanced order and in the same concentrations used in Experiment 1. Injections were delivered as in Experiment 


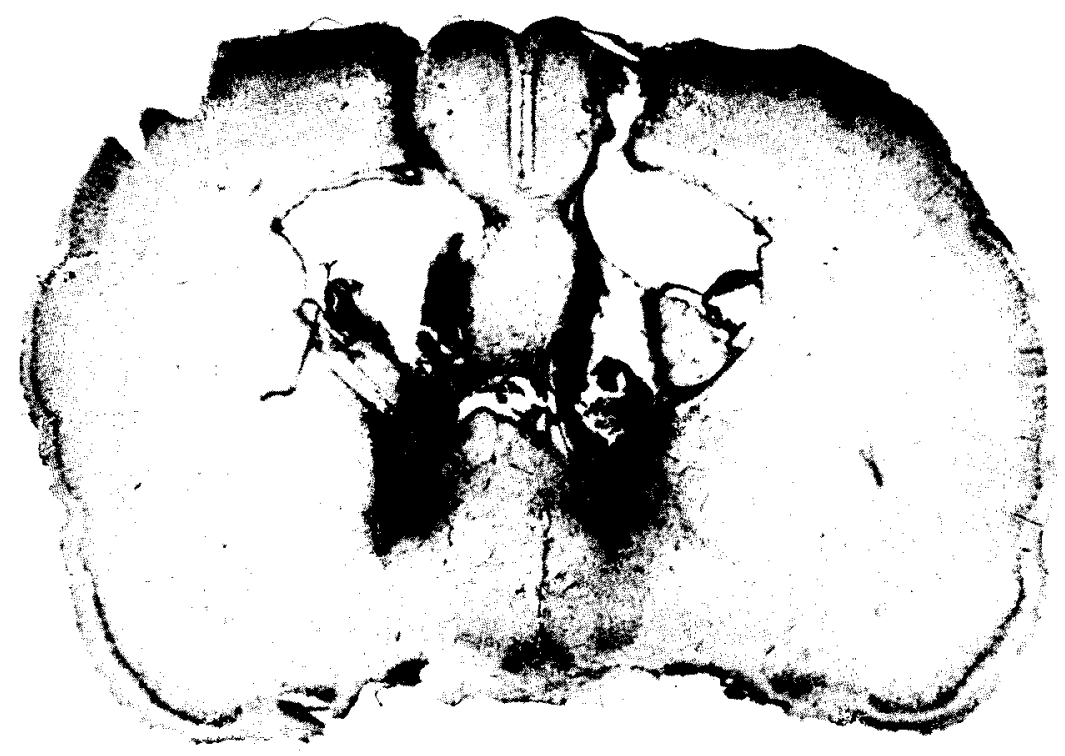

FIG. 1. Stained brain section from a rat in the $A D$ group of Experiment 1. Cannula tips are located in the interstitial nucleus of the stria terminalis.
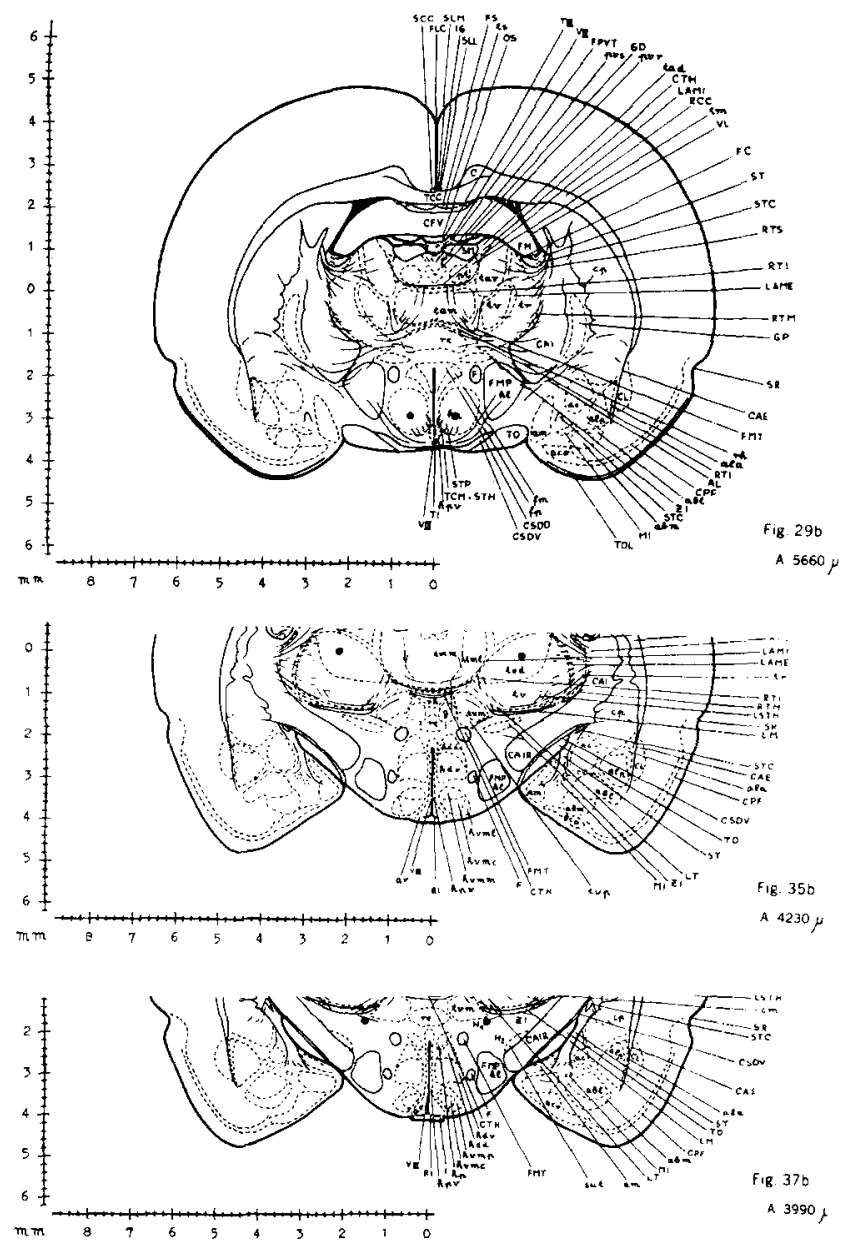

FIG. 2. Schematic representation showing other brain sites cannulated in Experiment 1. Filled circles represent placements in anterior hypothalamus, midthalamus, and the prerubal area. 
1 between the 30 and $60 \mathrm{~min}$ components of the satiated feeding schedule. Two l-NE injections were completed first, then $2 \mathrm{DA} \mathrm{HCl}$ injections interspersed with 4 saline injections during 8 consecutive days. Sixteen hour food intakes were also recorded following all central injections as in Experiment 1.

\section{Results}

Table 2 presents mean food intakes following l-NE and DA $\mathrm{HCl}$ injections for rats not injected; or injected with oil (Groups 1 and 2) or injected daily with $17 \beta$ estradiol (Group 3).

As can be seen from Table 2 norepinephrine significantly increased $(p<0.05)$ food intake for all groups irrespective of hormone conditions. Intracerebral dopamine $\mathrm{HCl}$ injections in the anterior diencephalon of rats not treated with estrogen (Groups 1 and 2), elicited significantly increased food intakes $(p<0.05)$ compared to saline controls. Conversely, for rats injected with daily subcutaneous doses of $17_{\beta}$ estradiol, dopamine $\mathrm{HCl}$ injections in the same anterior diencephalic locus resulted in a significant decrease $(p<0.05)$ in food intake.

Neither 1-NE nor DA $\mathrm{HCl}$ injections altered $16 \mathrm{hr}$ food intakes in the estradiol injected animals (Group 3). Sixteen hour food intakes following injection with $1-N E$ in the bined Groups 1 and 2 were, however, significantly decreased $t=-3.22(p<0.02)$ compared to similar intakes following saline injections. DA injections did not mirror this $1-\mathrm{NE}$ effect and resulted in no significant differences $(p>0.05)$ for $16 \mathrm{hr}$ food intakes.

The presence or absence of estrogen did not affect the degree of norepinephrine facilitated eating. Groups 1 and 2 were not significantly different $(p>0.05$, independent $t$ test) from Group 3. However, this was not the case following dopamine injections. Eating was facilitated only in the absence of estrogen. Food intakes were significantly greater ( $p<0.05$, independent $t$-test) for Groups 1 and 2 animals compared to Group 3 rats.

The efficacy of daily subcutaneous estradiol injections in the lowering of body weight is demonstrated by an analysis of body weights in noninjected rats and rats injected with sesame oil versus rats injected daily with $17 \beta$ estradiol. For animals in Groups 1 and 2 there was a net mean decrease of $6.6 \mathrm{~g}$ over the time course of the subcutaneous and central injections in this experiment. Initial and final body weights were not significantly different $(p>0.05)$. For rats in Group 3 , injected daily with $17 \beta$ estradiol, however, the mean decrease for this same period of time was $66.5 \mathrm{~g}$, and represented a significant body weight loss $(p<0.01)$.

\section{Histology}

Figure 3 shows a typical photograph from one rat cannulated in the INST. All animals had cannulas located in the substance of interstitial nucleus of the stria terminalis as localized by the König and Klippel atlas [6].

\section{DISCUSSION}

The significance of the observed results for a general theory of the regulation of food intake in the rat can only be evaluated by reference to the experimental hypothesis proposed in the introduction. To reiterate, it was postulated that estradiol inhibits $\beta$ dopamine hydroxlase in the dopamine to norepinephrine conversion thereby influencing norepinephrine availability at these rostral sites. Postulation

\section{TABLE 2}

MEAN FOOD INTAKES FOLLOWING I-NOREPINEPHRINE AND DOPAMINE HCI INJECTIONS IN OVARIECTOMIZED RATS CANNULATED IN THE ANTERIOR DIENCEPHALON WITH OR WITHOUT HORMONE REPLACEMENT

\begin{tabular}{llllc}
\hline & Mean & $\mathrm{N}$ & S. D. & $p$ \\
\hline $\begin{array}{l}\text { Norepinephrine } \\
\text { Groups 1 and 2 }\end{array}$ & +1.67 & 8 & 0.45 & $<0.05$ \\
Group 3 & +1.66 & 6 & 0.47 & $<0.05$ \\
& & & & \\
$\begin{array}{l}\text { Dopamine } \\
\text { Groups 1 and 2 }\end{array}$ & +3.40 & 7 & 0.82 & $<0.05$ \\
Group 3 & -1.30 & 5 & 0.55 & $<0.05$ \\
\hline
\end{tabular}

of this mechanism allowed, for the anterior diencephalon groups, differential predictions concerning the direction of the variations in the level of estradiol on food intake following norepinephrine and dopamine injections. Verification of the predicted feeding results during natural estrous and diestrous, following ovariectomy and with estradiol replacement for the anterior diencephalic groups provides support to the experimental hypothesis as a biochemical mechanism mediating catecholamine elicited eating in the female rat.

Inspection of Table 1 in Experiment 1 discloses an interesting result obtained with the catecholamine injections. While 1-NE injections in the anerior diencephalic group elicit increases during both phases of estrous cycle, the mean difference in intake during estrous is over twice as much as the increases elicited during diestrous. Conversely for dopamine, during estrous mean changes in food intake are many times less than changes elicited following dopamine injected during diestrous for the same rats. These observations may be at least indirect evidence suggesting the operation of a supersensitivity like mechanism at these anterior loci. A supersensitivity mechanism would be consistent with the experimental hypothesis which predicts increased amounts of 1-NE at diestrous but a lack of 1-NE during estrous. Another alternative interpretation of these differences might be the requirement for a functional pool of 1-NE at rostral sites necessary to mediate feeding behavior. Effects of 1-NE injections on feeding behavior would then depend upon how the additional l-NE affected this functional pool or its availability as recently suggested by Margules [7]. Alterations in the size and/or availability of this functional pool of norepinephrine could then be influenced by the endogenous levels of estradiol.

The efficacy of the hormone replacement therapy following overiectomy has been pointed out in the results. While the daily dose of exogenous estradiol may appear to be high, this dose was selected for two reasons: (a) the $17 \beta$ estradiol used was not in the benzoate form; and (b) Donoso and Stefano [2] demonstrated that this dose of estradiol reversed the levels of catecholamines in anterior hypothalamus following ovariectomy to those observed during estrous in the natural cycle. 


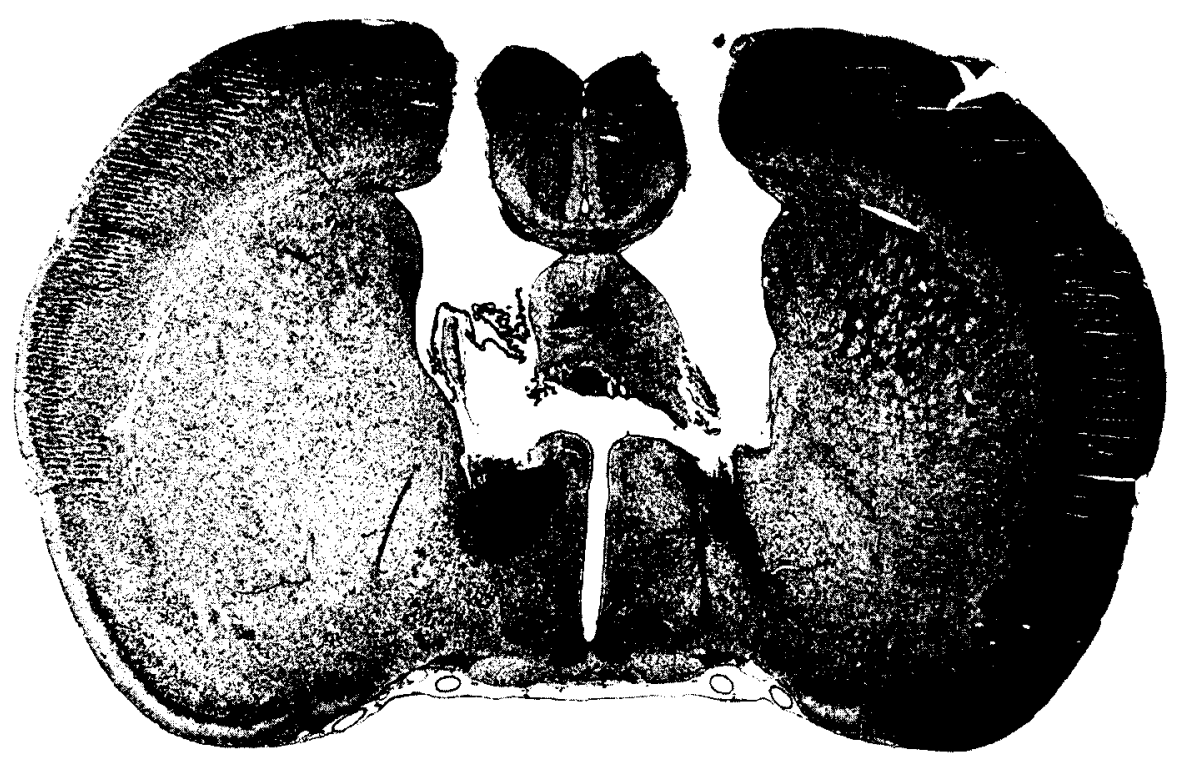

FIG. 3. Photograph of a brain section of a rat from Experiment 2. Cannula tips are located in the interstitial nucleus of the stria terminalis as localized by the König and Klippel atlas.

Food intake in one control group in Experiment 1 following catecholamine injections did produce some unexpected results. In the prerubal area, dopamine $\mathrm{HCl}$ injection elicited significant increases in food intake during diestrous. This effect has not previously been reported in the literature. The Fields of Forel are located anatomically in one of the efferent pathways from the lateral hypothalamus. Dopamine could be activating catecholamine coded efferent fibers involved with feeding responses organized in the lateral hypothalamus. One would expect, however, that norepinephrine should have similar effects when placed into the same area. Norepinephrine injections were without effect in producing changes in food intake when injected during diestrous. Dopamine elicited eating in the prerubal area is then an interesting phenomenon which should receive further investigation.

Intracranial catecholamine injections did not produce their effects on feeding behavior by disrupting the estrous cycle. Due to the fact that saline and catecholamine injections were counterbalanced in their order of presentation a number of rats received dopamine or norepinephrine injections for two successive days before the injection condition changed. Analysis of these records in terms of the stage of the estrous cycle, as determined from a daily vaginal smear, revealed no differences in the periodicity of cycling. That is, successive injections of either norepinephrine or dopamine did not sustain prolonged periods of either constant estrous or constant diestrous for any of the rats used in this study.

An alternative explanation, to account for the feeding results, suggested by Wade and Zucker [17] is that estrogens suppress food intake in adult female rats by their action on the ventromedial (VMH) nucleus of the hypothalamus. Wade and Zucker suggest that estrogens in adult females increase the activity of the VMH. Increased VMH activity then inhibits feeding responses organized in the lateral hypothalamus. Another possibility is that estrogens produce finicky eating behavior via the VMH which may be functionally equivalent to that seen in static hypothalamic hyperphagic rats.

Reynolds [9] has, however, examined the effects of estradiol replacement therapy on bilaterally ovariectomized female rats with electrolytic or radiofrequency lesions of the VMH. Consistent with Kakolewski, Cox and Valenstein [5] he found a significant increase in body weight gain following ovariectomy. Different groups of ovariectomized rats then received either radiofrequency or electrolytic lesions of the $\mathrm{VMH}$. Upon recovery from lesion surgery these rats received daily injections of estradiol or an oil vehicle. Estradiol replacement caused a significant decrease in the body weight of operated rats compared to oil injected controls. Reynolds' results support the idea that an intact VMH is not a necessary condition for the inhibitory effect of estradiol on food intake or body weight. Although estrogen influences on the VMH may be operative in the normal animal, Reynolds' results are consistent with the experimental hypothesis that estradiol inhibition of food intake occurs at hypothalamic loci other than the VMH.

The present experiments cannot evaluate the mechanism of estradiol influences on the VMH as proposed by Wade and Zucker [17]. Results have been presented, however, indicating that the estrogen inhibition of catecholamine elicited eating is not a result of disruption of the estrous cycle.

These studies support the hypothesis that estradiol inhibition of catecholamine elicited eating occurs at specific rostral brain sites and the mechanism of this inhibition is via $\beta$ dopamine hydroxlase. 


\section{REFERENCES}

1. Booth, D. A. Localization of the adrenergic feeding system in the rat diencephalon. Science 158: 515-517, 1967.

2. Donoso, A. O. and F. J. E. Stefano. Sex hormones and the concentration of noradrenaline and dopamine in the anterior hypothalamus of castrated rats. Experimentia 23: 665-666, 1965.

3. Donoso, A. O. and J. Cukier. Estrogen as depressor of noradrenalin concentration in the anterior hypothalamus. Nature 218: 969-970, 1968.

4. Donoso, A. P., M. B. deGuterrez Mayno and R. C. Santoleya. Metabolism of noradrenalin in hypothalamus of castrated rats. Neuroendocrinology 4: 72-79, 1969.

5. Kakolewski, J. W., V. C. Cox and E. S. Valenstein. Sex differences in body weight change following gonadectomy of rats. Psychol. Rep. 22: 547-554, 1968

6. König, J. F. R. and R. A. Klippel. The Rat Brain: A Stereotaxic Atlas. Baltimore, Maryland: The Williams and Wilkins Co., 1963.

7. Margules, D. L., M. J. Lewis, J. A. Dragovich and A. S. Margules. Hypothalamic norepinephrine: circadian rhythms and the control of feeding behavior. Science 178: 640-642, 1972.

8. Mook, D. G., N. J. Kenney, S. Roberts, A. I. Nussbaum and W. I. Rodier III. Ovarian-adrenal interactions in regulation of body weight by female rats. J. comp. physiol. Psychol. 81: 198-212, 1972.

9. Reynolds, R. W. Paper read at the Third International Conference on the Regulation of Food and Water Intake, Haverford College, 1968.

10. Simpson, C. W. Estradiol inhibition of catecholamine elicited eating in the female rat. Ph.D. Dissertation, U. C. Santa Barbara, April, 1970.
11. Slangen, J. H. and N. E. Miller. Pharmacological tests for the function of hypothalamic norepinephrine in eating behavior. Physiol. Behav. 4: 543-553, 1969.

12. Stefano, F. J. E. and A. O. Donoso. Norepinephrine levels in the rat hypothalamus during the estrous cycle. Endocrinology 81: $1405-1406,1967$.

13. Stumpf, W. E. Estrogen-neurons and Estrogen-neuron systems in the periventricular brain. Am. J. Anat. 129: 207-218, 1970.

14. Tartellin, M. F. and R. A. Gordki. Variations in food and water intake in the normal and acyclic female rat.Physiol. Behav. 7: $847-852,1971$.

15. Anton-Tay, F. and R. J. Wurtman. Norepinephrine: Turnover in rat brain after gonadectomy. Science 159: 1245, 1968.

16. Wade, G. M. and I. Zucker. Modulation of food intake and locomotor activity in female rats by diencephalic hormone implants. J. comp. physiol. Psychol. 72: 328-336, 1970.

17. Wade, G. M. and E. Zucker. Development of hormonal control over food intake and body weight in female rats. J. comp. physiol. Psychol. 70: 213-220, 1970.

18. Zarrow, M. X., J. M. Yochim and J. L. McCarthy. Experimental Endocrinology. A Sourcebook of Basic Techniques. New York: Academic Press, 1964, pp. 37-39.

19. Zucker, I. Body weight and age as factors determining Estrogen responsiveness in the rat feeding system. Behav. Biol. 7: $527-542,1972$.

20. Zucker, I. Hormonal determinants of sex differences in saccharin preference, food intake, and body weight. Physiol. Behav. 4: 595-602, 1969. 